\title{
Correlação entre Estadiamento Clínico e Cirúrgico de Pacientes com Carcinoma Invasor da Vulva
}

Correlation between Clinical and Surgical Staging of Patients with Invasive Vulvar Carcinoma

Angelo do Carmo Silva Matthes, Heitor Ricardo Cosiski Marana, Jurandyr Moreira da Andrade

Hélio Humberto Angotti Carrara, Francisco José Candido dos Reis

Maria Angeles Sanches Llorah Velludo, Sérgio Bighetti

\section{RESLMO}

Objetivos: avaliar a correlação entre o estadiamento clínico do câncer de vulva e o estadiamento cirúrgico, conforme a recomendação da Federação Internacional de Ginecologia e Obstetrícia (FIGO 95).

Pacientes e Métodos: foram estudados 66 casos de carcinoma invasor de vulva atendidos consecutivamente entre 1977 e 1997, em nosso Serviço. Todas as pacientes foram inicialmente estadiadas clinicamente, verificando-se o tamanho e a localização da lesão vulvar e o envolvimento ganglionar inguinal, bem como a extensão da lesão para outros órgãos. Destas 66 pacientes, 44 submeteram-se a tratamento cirúrgico, sendo que 34 puderam ser estadiadas conforme recomendação da FIGO 95.

Resultados: entre as 34 pacientes nas quais se realizou o estadiamento clínico e o cirúrgico, verificou-se 50\% (17 casos) de concordância e 50\% (17 casos) de discordância. Nos casos discordantes, o estádio cirúrgico foi superior em 13 (76,5\%) e inferior em 4 (23,5\%). O estádio clínico I foi reestadiado como estádio cirúrgico II em 2 casos $(22,2 \%)$ e III em 1 caso $(11,1 \%)$. O estádio clínico III tornou-se estádio cirúrgico I em 1 caso (12,5\%) e II em 3 casos (37,5\%). Conclusão: o estadiamento cirúrgico foi capaz de detectar casos de invasão ganglionar nãoverificados pelo exame clínico, assim como excluir casos falso-positivos. A concordância em apenas $50 \%$ dos casos mostra que o estadiamento clínico é insuficiente para pacientes com câncer da vulva.

PALAVRAS-CHAVE: Vulva: câncer. Câncer: estadiamento. Vulvectomia.

Introdução

O câncer da vulva (CV) constitui moléstia debilitante e freqüentemente é diagnosticado tardiamente, apesar de ser manifestação da genitália externa feminina. Em geral acomete pacientes do grupo etário avançado, dentre as quais 90\% encontram-se acima dos 50 anos $^{1}$, com um pico de incidência ao redor dos 66 anos $^{2}$, contudo, em torno de $12 \%$ a $15 \%$ dos casos ocorre em mulheres jovens, antes dos $45 \operatorname{anos}^{3,4}$. O pudor,

Departamento de Ginecologia e Obstetrícia da Faculdade de Medicina de Ribeirão Preto, Hospital das Clínicas Ribeirão Preto, USP

Correspondência:

Ângelo do Carmo Silva Matthes

Departamento de Ginecologia e Obstetrícia, Faculdade de Medicina de Ribeirão Preto, USP

Av. Bandeirantes, 3900 - $8^{\circ}$ andar

14049-900 - Ribeirão Preto - SP principalmente nas classes sociais menos favorecidas, pode retardar a procura do médico pela paciente ${ }^{1}$. Em alguns casos, pode ocorrer também falha na avaliação, quando o tumor está em sua fase incipiente ${ }^{2}$. Nos países em desenvolvimento, o diagnóstico freqüentemente é feito em estádios mais avançados ${ }^{5}$, chegando a $84 \%$ dos casos nos estádios III e IV ${ }^{6}$. Nos países desenvolvidos o diagnóstico nos estádios III e IV oscila entre 18,5 e $49 \%{ }^{7}$ e, no Brasil, a descoberta de casos nos estádios III e IV ocorre em $36 \%$ a $57 \%$ das pacientes ${ }^{8,9}$.

O fator prognóstico mais importante é o acometimento dos linfonodos regionais. A sobrevida pode chegar a 90\% quando os linfonodos são negativos, independente do estádio inicial, sofrendo redução para $25 \%$ a $60 \%$ quando estes linfonodos são positivos ${ }^{3,10,11}$. Alguns autores têm insistido que a sobrevida de pacientes com câncer da vulva depende do estadiamento recomendado pela FIGO, 
esperando-se 96 a 100\% de sobrevida em 5 anos no estádio I, ao passo que para pacientes com tumores no estadio IV, esta seria de 19 a 36\% ${ }^{5,12}$.

As orientações do Comitê de Câncer da FIGO anteriores a 1988 eram para que o estadiamento clínico obedecesse à classificação TNM da UICC. Para o câncer da vulva, no entanto, esta classificação apresenta uma séria limitação, pois depende de uma medida arbitrária de $2 \mathrm{~cm}$ do tumor primário, sem considerar lesões maiores, resultando em uma distribuição desigual de pacientes com diagnósticos imprecisos nos tumores localmente avançados e com envolvimento linfonodal regional. A palpação clínica dos linfonodos é limitada para detectar metástases e por isso o estadiamento cirúrgico é mais adequado, ao oferecer uma avaliação histológica destes, permitindo estabelecer um melhor prognóstico e recuperar dados ${ }^{13}$.

As orientações da FIGO de 1988, ratificadas em 1995, recomendam o estadiamento cirúrgicopatológico, o que implica comprovação histopatológica de comprometimento dos gânglios inguinais. Assim, o estadio N1 corresponde à presença de gânglios metastáticos unilaterais, representando o estadio III; N2 corresponde à presença de metástases ganglionares bilaterais, representando o estadio IVa, e M1 corresponde ao comprometimento de gânglios pélvicos, representando o estadio $\mathrm{IVb}$.

Buscou-se neste trabalho verificar a correlação entre o estadiamento clínico inicial no diagnóstico da doença com o verificado após a cirurgia para tratamento das pacientes com $\mathrm{CV}$ assistidas em nosso Serviço.

\section{Pacientes e Métodos}

Foram estudados os casos de $\mathrm{CV}$ atendidos entre 1977 e 1997, no Setor de Oncologia Ginecológica do Departamento de Ginecologia e Obstetrícia do Hospital das Clínicas da Faculdade de Medicina de Ribeirão Preto da Universidade de São Paulo.

Neste período foram tratadas 66 pacientes com diagnóstico de câncer invasor operável e que tiveram atendimento inicial no serviço. Procedeuse à confecção de uma ficha de investigação na qual se anotaram o estadiamento clínico quando do diagnóstico, o tratamento realizado, o resultado do estudo anatomopatológico pós-cirúrgico, tempo de evolução e condição física no último retorno. A partir destes dados foi avaliada a correlação clínicocirúrgica dos estadiamentos e sua repercussão na sobrevida e no intervalo livre de doença. Além disto, analisamos para este grupo de pacientes alguns fatores prognósticos como a idade, o grau histológico, a associação com outras lesões vulvares e o tipo de tratamento.

\section{Resultados}

As 66 pacientes incluídas neste estudo tinham idade entre 27 e 87 anos, com média e mediana de 67,5 anos com 3 modas de 4 pacientes com 60, 76 e 80 anos, respectivamente. Somente 10 pacientes apresentavam idade igual ou inferior a 50 anos. As 34 pacientes que foram estadiadas cirurgicamente tinham média de idade de 65 anos e mediana de 67 anos. A maioria das pacientes desta casuística encontravam-se nos estádios iniciais, dos quais $57,6 \%$ dos casos nos estádios clínicos I e II e 55,9\% nos estádios cirúrgicos I e II (Tabela 1).

Tabela 1 - Freqüência de pacientes com câncer de vulva, segundo estadiamento clínico e cirúrgico.

Estádio Estadiamento clínico (\%) Estadiamento cirúrgico (\%)

$\begin{array}{lrr}\text { I } & 16,7 & 20,6 \\ \text { II } & 40,9 & 35,3 \\ \text { III } & 22,7 & 26,5 \\ \text { IVa } & 16,7 & 14,7 \\ \text { IVb } & 3,0 & 2,9\end{array}$

Para o grupo de 34 pacientes submetidas a vulvectomia com linfadenectomia inguinal superficial e profunda bilateral, pudemos estabelecer uma correlação entre o estádio clínico e cirúrgico. Observamos que em 17 oportunidades o estádio clínico correspondeu ao cirúrgico. Em 13 vezes o estadiamento cirúrgico foi superior ao clínico e em 4 vezes ocorreu o inverso (Tabela 2). Das 14 pacientes tratadas com vulvectomia radical e linfadenectomia pélvica, 1 caso apresentou linfonodos positivos. Neste grupo, 6 pacientes foram classificadas no estádio II, 7 no III e 1 no IVb.

Tabela 2 - Correlação entre o estadiamento clínico e o cirúrgico em 34 pacientes com câncer de vulva.

\begin{tabular}{|c|c|c|c|c|c|c|}
\hline \multirow[t]{2}{*}{ Clínico } & \multicolumn{6}{|c|}{ Cirúrgico } \\
\hline & I & II & III & $\mathrm{IVa}$ & $\mathrm{IVb}$ & Total \\
\hline I & 6 & 2 & 1 & & & 9 \\
\hline$\|$ & & 7 & 6 & 1 & 1 & 15 \\
\hline III & 1 & 3 & 2 & 2 & & 8 \\
\hline IVa & & & & 2 & & 2 \\
\hline $\mathrm{IVb}$ & & & & & 0 & 0 \\
\hline Total & 7 & 12 & 9 & 5 & 1 & 34 \\
\hline
\end{tabular}


Entre as 34 pacientes tratadas cirurgicamente, em 30 utilizou-se incisão única, retirando-se a peça em monobloco, e em quatro empregou-se a técnica de 3 incisões.

\section{Discussão}

As pacientes com idade superior a 50 anos representaram $85 \%$ da amostra estudada, que tem como idade média 67,5 anos, o que concorda com outras casuísticas que indicam de 65 a 69 $\operatorname{anos}^{11,12,14,15}$. Torres Lobaton et al. ${ }^{11}$ relatam o caso da paciente mais jovem, com 14 anos. No presente estudo, a paciente mais jovem tinha 27 anos e 10 apresentaram idade inferior a 50 anos, o que representou $15 \%$ dos casos. Porcentagem idêntica foi referida por Henson e Tarone ${ }^{16}$. Apesar de o câncer da vulva ser mais comum em mulheres idosas ${ }^{17}$, tem sido relatado um aumento de sua incidência em mulheres mais jovens ${ }^{18,19}$.

Para a correlação entre os estádios clínico e cirúrgico seguimos a orientação da FIGO para o estadiamento clínico, tendo sido avaliados o envolvimento dos linfonodos, o tamanho do tumor e o comprometimento de órgãos vizinhos. Assim, $83,3 \%$ das nossas pacientes foram incluídas nos estádios II, III e IV e 16,7\% no estádio I, dados também semelhantes aos de outros autores ${ }^{9,13}$, mas diferentes dos de Rosen e Malmström ${ }^{12}$, que classificaram $35 \%$ das suas pacientes no estádio I. O motivo da predominância de estadios avançados entre os nossos casos pode ser explicado pela demora em se procurar assistência médica ${ }^{20}$.

Em 34 pacientes foi possivel realizar o estadiamento cirúrgico, havendo concordância com o estadiamento clínico prévio em 50\% dos casos. Houve um aumento na porcentagem de pacientes no estadio I, após o estadiamento cirúrgico, provavelmente por ter o estadiamento clínico caracterizado erroneamente linfonodos como metastáticos, quando eram inflamatórios. O estádio I correspondeu a $20,6 \%$ dos casos estadiados cirurgicamente e Origoni et al. ${ }^{10}$ relatam 16,9\% de estádio I cirúrgico. Na outra metade em que não houve correspondência, verificou-se que o estádio cirúrgico foi superior em 13 vezes e inferior em 4. Isto implica que ocorreu erro de estadiamento em 38\% destas pacientes, presumindo-se ser o tumor menos avançado do que na realidade se apresentava. No entanto, somente entre os casos do estádio I este fato pôde interferir no tratamento. Em nosso material o estádio I clínico foi modificado para II em 2 casos e III em 1, não havendo relato prévio na literatura deste tipo de erro de avaliação, provavelmente por uma falha clínica da avaliação linfonodal inguinal.

O tratamento de eleição para o câncer da vulva é o cirúrgico. Não há esquema rígido de tratamento para todos os casos, sendo limitantes fatores orgânicos e a negação da paciente em se submeter a uma cirurgia mutilante e deformante ${ }^{21}$. Em 14 pacientes realizamos vulvectomia com linfadenectomia inguinal e pélvica. $\mathrm{O}$ exame dos linfonodos pélvicos foram negativos, exceto em 1 caso $(7,1 \%)$. Este achado implica admitir que estas pacientes foram sobretratadas. Neste grupo 6 pacientes foram estadiadas como II, 7 como III e 1 como IVb, o que torna uma cirurgia com esta extensão uma exceção para casos extremamente selecionados, podendo a linfadenectomia pélvica ser substituída pela radioterapia.

Em 30 cirurgias a incisão foi única, retirandose a peça em monobloco, e em 4 empregamos 3 incisões. Verdiani et al. ${ }^{15}$, relatam 132 casos tratados cirurgicamente, dos quais $65(49 \%)$ por incisão única e 67 (51\%) por 3 incisões concluindo que as complicações pós-operatórias e a necessidade de procedimentos secundários foram significativamente menos freqüentes nas pacientes submetidas à cirurgia com 3 incisões, preconizando seu uso nas vulvectomias.

Concluímos que, em paciente com câncer da vulva, o estadiamento clínico deve ser considerado provisório, embora haja boa correlação entre o estadiamento clínico e cirúrgico. Por outro lado, a linfadenectomia pélvica deve ser evitada, pela baixa freqüência de linfonodos com metástases, podendo ser substituída pela radioterapia pélvica em casos selecionados.

\section{SUMMARY}

Purpose: to evaluate the correlation between clinical and surgical staging of patients with vulvar carcinoma, according to the "Federação Internacional de Ginecologia $e$ Obstetrícia” (FIGO 95).

Methods: the authors studied 66 consecutive cases of vulvar carcinoma from 1977 to 1997. All patients were clinically staged to verify size and localization of the lesion as well as inguinal lymph node involvement and invasion of other organs. Forty-four patients were submitted to surgical treatment and 34 could be staged according to FIGO 95.

Results: among the 34 patients staged through surgery, 17 (50\%) showed agreement between clinical and surgical staging. Thus, in 17 patients the staging was different and in these the surgical staging was higher than the clinical in 13 cases and lower in 4 cases. We found in the clinical staging I, 2 cases that were surgical stage II and 1 case that was surgical stage III.

Conclusion: the surgical staging could detect lymph node metastasis in patients with clinically negative nodes, as well as exclude false-positive cases. The clinical staging was not accurate for patients with vulvar carcinoma.

KEY WORDS: Vulvar carcinoma. Surgical staging. Vulvectomy. 


\section{Referências}

1. Abrão FS, Abrão MS. Câncer da vulva - diagnóstico, epidemiologia e estadiamento. In: Abrão FS, et al., editor. Tratado de Oncologia Genital e Mamária. São Paulo: Roca; 1995. p.474-87.

2. Girão MJC, Endo RM, Sartori MGF, Kosmiskas JVC, Gonçalves WJ, Baracat EC, et al. Câncer da vulva - Estudo epidemiológico. Ginecol Obstet Atual 1995; $1:$ 42-4.

3. Disaia PJ, Creasman WM. Invasive cancer of the vulva. In: DiSaia PAJ, Creasman WT, editores. Clinical Gynecology Oncology. 3 ed. St Louis: Mosby; 1989. p.241-72.

4. Nilsson T, Alm P, Malmström H, Simonsen E, Tropé C. A 16-year-old girl with invasive carcinoma in the vulva. Acta Obstet Gynecol Scand 1990; 69: 541-2.

5. Perez CA, Grigsby RW. Vulva. In: Perez CA, Brady LW, editor. Principles and Practice of Radiation Gynecologic Oncology. Philadephia: Lippincott; 1992. p.1273-89.

6. Torres Lobaton A, Mendoza Tenorio A, Palacios Castillo A. Tratamiento quirúrgico del cáncer de la vulva. Experiencia con 43 casos. Ginecol Obstet Méx 1983; 51: 287-96.

7. Shanbour KA, Mannel RS, Morris PC, Yadack A, Walker JL. Comparison of clinical versus surgical staging systems in vulvar cancer. Obstet Gynecol 1992; 80: 927-30.

8. Abrão FS, Baracat EC, Marques AF, Abrão MS, Torloni $\mathrm{H}$, Coelho FR, et al. Carcinoma of the vulva. Clinicopathologic factors involved in inguinal and pelvic lymph node metastasis. J Reprod Med 1990; 35: 1113-6.

9. Souen JS, Filassi JR, Chin HC, Motta EV. Câncer da vulva na clinica ginecológica da Faculdade de Medicina da Universidade de São Paulo. Rev Bras Ginecol Obstet 1992; 14: 87-92.

10. Origoni M, Dindelli M, Ferrari D, Frigerio L, Rossi M, Ferrari A. Surgical staging of invasive squamous cell carcinoma of the vulva. Analysis of treatment and survival. Int Surg 1996; 81: 67-70.
11.Torres Lobaton A, Plata Nuñez P, Roman Bassaure E, Hernandez Aten, D, Garcia Gonzalez H. Neoplasias malignas de la vulva (análisis de 113 pacientes). Ginecol Obstet Méx 1989; 57: 235-41.

12.Rosen C, Malmstrom $\mathrm{H}$. Invasive cancer of the vulva. Gynecol Oncol 1997; 65: 213-7.

13.Krupp Jr PJ. Invasive tumors of vulva: clinical features, staging and management. In: Coppleson M, Monaghan JM, Morrow CP, Tattersall MHM, editores. Gynecologic Oncology: Fundamental Principles and Clinical Practice. 2 ed. New York: Churchill Livingstone; 1992. p. 479-91.

14.Smyczek-Gargya B, Volz B, Geppert M, Dietl J. A multivariate analysis of clinical and morphological prognostic factors in squamous cell carcinoma of the vulva. Gynecol Obstet Invest 1997; 43: 261-7.

15.Verdiani LA, Derchain SFM, Juliato CRT. Vulvar carcinoma epidemiology and clinical aspects. In: Convenção Latino-Americana da European School of Oncology, São Paulo, Abstrats. São Paulo: Comitê Brasileiro da ESO; 1997. (Abstract, 29-B2).

16.Henson D, Tarone R. An epidemiologic study of cancer of the cervix, vagina and vulva based on the Third National Cancer Survey in the United States. Am J Obstet Ginecol 1977; 129: 525-32.

17.Tuffi VHB, Eluf Neto J, Marques JA, Carvalho FM, Souen JS. Carcinoma de vulva. Rev Ginecol Obstet 1997; 8: 113-8.

18.Andersen WA, Franquemont DW, Williams J, Taylor PT, Crum CP. Vulvar squamous cell carcinoma and papillomaviruses: two separate entities? Am J Obstet Gynecol 1991; 165: 329-36.

19. Roman LD, Mitchell MF, Burke TW, Silva EG. Unsuspected invasive squamous cell carcinoma of the vulva in young women. Gynecol Oncol $1991 ; 41: 182-5$.

20.Noreña GG. Incidencia del cancer de vulva. Hospital Universitario San Vicent de Paul 1972 - 1982. Rev Colomb Obstet Ginecol 1982; 33: 257-66.

21.Abrão FS, Marziona F, Coelho FRG, Chazan R, Abrão M. Câncer da vulva: tratamento cirúrgico e complicações. Femina 1991; 19: 964-6. 